\title{
Developed Forward and Inverse Modelling of Vertical Electrical Sounding (VES) Using MATLAB Implementation
}

\author{
La Hamimu ${ }^{1}$, Jamhir Safani ${ }^{2}$, LO Ngkoimani ${ }^{3}$ \\ ${ }^{1,2,3}$ Department of Geophysical Engineering, Unversitas Halu Oleo, Kendari (93232), South East Sulawesi, Indonesia
}

\begin{abstract}
We have implemented a MATLAB source code for VES forward modeling and its inversion using a genetic algorithm (GA) optimization technique. The codes presented here are applied to the Schlumberger electrode arrangement. In the forward modeling computation, we have developed code to generate theoretical apparent resistivity curves from a specified layered earth model. The input to this program consists of the number of layers, the layer resistivity and thickness. The output of this program is apparent resistivity versus electrode spacing incorporated in the inversion process as apparent resistivity data. For the inversion, we have developed a MATLAB code to invert (for layer resistivity and thickness) the apparent resistivity data by the genetic algorithm optimization technique. The code also has some function files involving the basic stages in the GA inversion. Our inversion procedure addressed calculates forward solutions from sets of random input, to find the apparent resistivity. Then, it evolves the models by better sets of inputs through processes that imitate natural mating, selection, crossover, and mutation in each generation. The aim of GA inversion is to find the best correlation between model and theoretical apparent resistivity curves. In this study, we present three synthetic examples that demonstrate the effectiveness and usefulness of this program. Our numerical modeling shows that the GA optimization technique can be applied for resolving layer parameters with reasonably low error values.
\end{abstract}

Keywords: Forward and inverse modelling, vertical electrical sounding, MATLAB implementation, genetic algorithm, apparent resistivity

\section{Introduction}

A great variety of computer programs and software products have been developed for resistivity modeling and inversion algorithm due to very fast enhancement in computers and technology. Albeit that 2D and 3D modeling and inversion algorithms such as RES2DINV software and RES3DINV software are now available; $2 \mathrm{D}$ and $3 \mathrm{D}$ resistivity techniques for data acquisition that provide adequate coverage are generally very expensive; and 3D numerical modeling and inversion algorithms need more computation time and are also more time-consuming and elaborate for field data interpretation. As a consequence, many resistivity surveys are still being done with $1 \mathrm{D}$ resistivity or VES inversion still playing an important role for initial analysis in field applications. When the resistivity changes are smooth, 1D resistivity surveys can easily be used to interpret the data [1].

In the last few decades, many papers have been extensively published on various methods to invert apparent resistivity data. For the 1D resistivity inversion problem, many authors employ linear inversion such as Marquradt's algorithm [2], iterative least square procedure (ILSQP) with singular value decomposition (SVD) [1], least square method with ridge regression [3], and Occam's inversion algorithm [4]. These linearized methods are considered as an acceptable and computational effective solution when some robust a priori information and good starting models are available. In general, the apparent resistivity values measured on the surface as a function of electrode separation bear a nonlinear relationship to the model parameters, i.e., the resistivity and thickness of layers comprising a 1D earth model. The use of nonlinear optimization techniques such as simulated annealing and genetic algorithm are therefore a natural choice to solve geophysical inverse problems such as phase velocity dispersion curves and apparent resistivity [5].

In recent years there has been increasing use of genetic algorithms in solving geophysical inverse problem (e.g. Nagai et al., [6], Song and Gu, [7], Hamimu et al., [8, 9]). These authors have used GA optimization techniques for inversion of surface wave dispersion curves. The promising results obtained so far suggest that GA can be successfully applied to other areas of geophysics, including resistivity inverse problems. For example, Jha et al., [10] developed GA optimization technique for resistivity inversion from vertical electrical sounding (VES) using computer code in ' $\mathrm{C}$ ' programming language. In this paper, we provide and test Matlab code that calculates an inversion of the apparent resistivity of a layered earth in VES.

In most cases, VES forward modeling and GA inversion codes which are available in the public domain are generally coded in FORTRAN or the $\mathrm{C}$ programming language (e.g. Davis [2], Nagai et al., [6]; Jha et al., [10]; Kohlbeck and Mawlood [11], Hamimu et al., [8, 9]. However, FORTRAN and $\mathrm{C}$ programming languages impose a rather expensive burden on users in integrating complicated numerical algorithms with visual data analysis compared with MATLAB, which provides integrated functionality of computation and visualization. MATLAB is a powerful script programming language and computation environment in that it provides extensive numerical libraries, data visualization capabilities, and enables the user to interactively exploit data variables step by step during execution. Matlab employs a high-level programming language grammar, resulting from simple vector and matrix multiplication based on arrays that need no memory dimensioning. It also allows one to have the benefit of using object oriented programming [12].

\section{Volume 4 Issue 11, November 2015}




\section{International Journal of Science and Research (IJSR) \\ ISSN (Online): 2319-7064}

Index Copernicus Value (2013): 6.14 | Impact Factor (2014): 5.611

In this paper, we present novel program codes written in the MATLAB programming language for solution to the forward and inversion problems of VES using a Schlumberger array. The codes presented here compute a forward modeling of the Schlumberger array in order to generate the theoretical apparent resistivity values at different electrode spacings. The main intention of this study is to develop computational codes for modelling and inversion of apparent resistivity data using GA optimization technique. All computation processes are implemented under the MATLAB environment using version 8.4.0.150421 Release 2014b. The developed codes are also tested using two synthetic examples that demonstrate their robustness and usefulness.

\section{Forward Modeling of VES}

The relation between the resistivity transform and the layer distribution was first described by Ghosh [13]. In his paper, a fast method was developed for computing the apparent resistivity curves from a known layer configuration of thickness and resistivity. The method is based on the application of a linear filter to determine the apparent resistivity curve from the kernel function. The apparent resistivity curve is obtained in two stages: the following steps are incorporated in the computation of apparent resistivity curves by Ghosh's inverse filter method. In the first stage, the sample values of the resistivity transform function are obtained for a given model. This is accomplished by the application of Pekeris recurrence relation to calculate the resistivity function, $T(m)$, for a multi-layer ( $N$ layers) subsurface system, which is given as:

$$
T_{i}=\frac{\left[T_{i+1}+\rho_{i} \tanh \left(m h_{i}\right)\right]}{\left[1+T_{i+1} \tanh \left(m h_{i}\right) / \rho_{i}\right]}
$$

where $T_{N}$ is $\rho_{N}$ for the lowermost layer and $T_{1}$ corresponds to $T(m)$ at the earth's surface, $\rho_{i}$ is the resistivity of the $i$ th layer, and $h_{i}$ the thickness of the $i$ th layer. In the second stage the transform sample values are convolved with the filter coefficients $b_{j}$ to generate the apparent resistivity values $R_{m}$. The resistivity transform function can be extracted from the apparent resistivity function by the application of simple linear filter using Ghosh [13] formula:

$$
T_{m}=\sum_{j=-\infty}^{j=\infty} b_{j} R_{m-j}
$$

where $T_{m}$ is the resistivity transform at sample point $m, m$ is the determined number of sample points, $b_{j}$ is the filter coefficients, number by length of the filter to be used and $R_{m}$ is the apparent resistivity value at sample point $m$. These equations are used to solve the forward problem.

The forward problem consists of computing an apparent resistivity curve for some theoretical model. This is normally accomplished by the RESIST program using linear filter theory [13]. The RESIST program, described by Davis [2], is programmed in FORTRAN. In this study, we adopted and modified Davis's program to develop a forward modeling of VES using the MATLAB programming language. In the forward modeling program, two M-files of MATLAB are created for computing experimental apparent resistivity values from a specified layered earth model. The input to the forward program comprises the subsurface parameters for a layered earth model. These include: the number of layers, the resistivity values of each layer, the layer thicknesses and the electrode spacing. The output from the forward modelling function yields a curve of apparent resistivity versus electrode spacing.

\section{Inversion Using the GA Optimization Technique}

In this study, inversion of apparent resistivity is developed by adopting the FORTRAN GA inversion codes of Hamimu et al., $[8,9]$ which were used for inverting surface wave phase velocity curves. For 1D resistivity inversion, GA inversion seeks to find the best combination of resistivity and thickness values for each layer to minimize the difference between the experimental and the theoretical apparent resistivity curve. The forward program used to calculate the theoretical apparent resistivity for the inverse procedure is based on the RESIST program of Davis [13], and the root mean square error $\left(E_{r m s}\right)$ considered is an objective function to be minimized expressed as follows:

$$
E_{r m s}=\sqrt{\frac{1}{N} \sum_{k=1}^{N}\left(\rho o^{k}-\rho i^{k}\right)^{2}}
$$

where $\rho o^{k}$ is the observed apparent resistivity curve of the $k$ th data point, $\rho i^{k}$ is the inverted apparent resistivity curve of the $k$ th data point, and $N$ is the total number of field spacings desired. The optimization problem addressed in GA inversion is also subjected to the following constraints:

$$
\rho^{\min } \leq \rho \leq \rho^{\max } ; h^{\min } \leq h \leq h^{\max }
$$

where $\rho^{\min }$ and $\rho^{\max }$ are vectors of the lower and upper bound assigned to each layer's resistivity, respectively; and $h^{\min }$ and $h^{\max }$ are vectors of the lower and upper bound on the thickness of each layer, respectively.

In GA inversion, resistivity values and thicknesses of the layers are the parameters to be determined. These parameters are encoded in gene type that appropriate for the genetic operations. We refer to Sen and Stoffa [14] for coding inversion parameters. First, we define search limits for the apparent resistivity and thickness for each layer. Each parameter in the search area is digitized with an 8-bit binary string of $2^{8}$, that is, parameters at the lower and upper limit became $(00 \ldots 0)$ and $(11 \ldots 1)$, respectively, and any parameter to be examined could then be transferred in a binary string between the two bit strings. The binary strings for all parameters in a subsurface structural model were concatenated to generate a chromosome to be used in the genetic operation.

Using random number, we generate an initial population that consists of 64 individuals, i.e., 64 different models. Then, the three genetic operators (selection, crossover and mutation) are applied to the initial population to produce a new population with same population size. The entire process is randomized, starting with the initial parent populations, and the selection-crossover-mutation of each bit string at each iteration. The algorithm we implemented is based on the forking GA (fGA) of Tsutsui et al., [15]. This employs a kind of elitism, which selectively searches parts of the multi-

\section{Volume 4 Issue 11, November 2015}




\section{International Journal of Science and Research (IJSR) \\ ISSN (Online): 2319-7064}

Index Copernicus Value (2013): 6.14 | Impact Factor (2014): 5.611

population search spaces, avoiding local minima. The search space is divided using information based on the convergence status of the populations, known as a phenotypic fGA.

In selection, a new population is reproduced based on fitness function (Equation 3) for each individual. A mathematical expression which is called as a fitness function is used to calculate a value for a solution of the objective function. The fitter solution gets the higher value and the ones (other solutions) that violate the objective function and constraints are penalized. When the model has a low misfit function, it has high probability of being reproduced and passed down in the next generation. Therefore, like in nature, the model having the highest fitness and best solutions will survive and get the chance to be selected for inclusion in the next generation.

In crossover, all surviving individuals are randomly paired. Crossover is a recombination operator that proceeds in three steps [16]: (a) the reproduction operator selects at random a pair of two model strings for mating, (b) a cross site is selected at random along the string length, and (c) the position values are swapped between the two strings following the cross site. At a given crossover probability $\left(p_{c}\right)$, the pair exchange a part of each bit string corresponding to each model parameter at a random locus in the string. In the calculation, a random number ranging from 0.0 to 1.0 is generated. Then, if the generated random number was smaller than crossover probability, the crossover process took place, and two new individuals were created and replaced the previous individual.

In mutation, genes in each chromosome randomly change from 0 to 1 or vice versa. Similar to crossover, we determine the occurrence of the mutation by generating random numbers. In our case study, we set the mutation probability at a high mutation of 0.2 . This high mutation probability results in a large number of random walks, but can also delay convergence of the algorithm to the optimally fit model. Therefore in our inversion procedures, the optimization is allowed to run for a maximum number of iteration and no other regularization, such as termination below a certain $E_{r m s}$ value or when the population became homogeneous, is applied. The algorithm is terminated at the $100^{\text {th }}$ iteration. By iteration of these three genetic operations, the initial population approaches a global optimal solution. Genetic algorithm is one of the kinds of probabilistic inversion method using random number and searches models near global minimum. Therefore 20 inversions are incorporated, where each inversion has same input parameters but different initial random numbers. A final optimal model was found out by averaging the resulting parameters from these 20 inversions [17].

In these works, a number of $\mathrm{M}$-file codes involving four basic steps in GA have been successfully made. The following description is a brief outline of how the different genetic operators are coded in the different M-files.

\subsection{Generating the first population}

In this step, an initial population of the parameters, namely a set of solutions, proportional to the total length of string is generated employing a random generator. Each population is then presented by a set of parameter values that illustrate the problem. A MATLAB function file called as call_firstpop.m file is generated to produce the initial populations employing population forking method of Tsutsui et al., [15]. By using random number, an initial population consists of 64 individuals, i.e., 64 different models, can be randomly generated.

\subsection{Coding the representation of model parameters}

For coding inversion parameters, the search area of the resistivity and thickness values for each layer are first defined. This coding has been realized by a MATLAB function file call_decod.m. It also encodes the model parameters in a binary string. For example, consider a problem where different geologic unit have different physical properties and these properties represent the model parameter to be estimated. The resistivity values can be considered as an example of physical properties to be coded.

\subsection{Selection based on an objective function}

In selection a group of models is chosen at random from the population, and the model having the highest fitness is selected for inclusion in the next generation. This fitness value (determined by means of an objective function) is then considered in the successive selection and this procedure is repeated until an appropriate number of models are selected for the new generation. A MATLAB function file which is called call_calfit.m file is developed to calculate fitness values. In this step the root mean square error $\left(E_{r m s}\right)$ (equation 3 ) is considered as an objective function.

\subsection{Crossover and mutation operators}

Crossover is the mechanism that allows genetic information between the paired models to be shared. It causes the exchange of some information between the paired models, thereby generating new pairs model. Mutation, which is another important operator, is the random alteration of a bit. It can be carried out during the crossover process. A MATLAB function file called call_crossmut.m written for implementing crossover and mutation operators. The number of strings in which the pair model is exchanged is controlled by the crossover probability $\left(p_{c}\right)$. The mutation rate is also specified by a mutation probability $\left(p_{m}\right)$. In this study, $p_{c}$ and $p_{m}$ are all set up at 0.2 .

\section{Numerical Error}

To estimate the accuracy of our GA inversion codes, differences between the inverted values of model parameters and the true ones are quantified in terms of the resistivity values error $\left(E_{r}\right)$ and the thickness error $\left(E_{h}\right)$, respectively. The resistivity and thickness errors are a measure of total error between the inverted and true of the formation resistivity and thicknesses, and are defined as following:

\section{Volume 4 Issue 11, November 2015}


International Journal of Science and Research (IJSR)

ISSN (Online): 2319-7064

Index Copernicus Value (2013): 6.14 | Impact Factor (2014): 5.611

$$
\begin{aligned}
& E_{r}=100 \% \times\left\{\sum_{k=1}^{M}\left[\left(\rho i^{k}-\rho t^{k}\right)^{2} /\left(\rho t^{k}\right)^{2}\right]\right\}^{1 / 2} \\
& E_{h}=100 \% \times\left\{\sum_{k=1}^{M}\left[\left(h i^{k}-h t^{k}\right)^{2} /\left(h t^{k}\right)^{2}\right]\right\}^{1 / 2}
\end{aligned}
$$

where $\rho i^{k}$ is the inverted resistivity value and $\rho t^{k}$ is the true resistivity value, $h i^{k}$ is the inverted thickness and $h t^{k}$ is the true thickness of the $k$ th layer, respectively. The variable $M$ is the number of layers.

A second measure of error, relative resistivity value and thickness errors for each layer was also calculated to investigate the deviation of inversion results for each layer. These relative errors are defined as

$$
E R_{k}=\left(\frac{\left|\rho i^{k}-\rho t^{k}\right|}{\rho t^{k}}\right) ; E H_{k}=\left(\frac{\left|h i^{k}-h t^{k}\right|}{h t^{k}}\right)
$$

where $E R_{k}=$ relative resistivity value error for $k$ th layer and $E H_{k}=$ relative thickness error for $k$ th layer.

The last measure of error, the inverted and observed resistivity curve errors $\left(E_{\rho}\right)$, was also calculated and used to assess the effectiveness of the GA inversion. An $E_{\rho}$ is a measure of the difference between the inverted apparent resistivity curve, which is the best inversion result, and the observed one, and is defined as following:

$$
E_{\rho}=\sqrt{\frac{1}{N} \sum_{k=1}^{N}\left(\rho i^{k}-\rho t^{k}\right)^{2}}
$$

where $\rho i^{k}$ is the theoretical apparent resistivity curve obtained from the forward modeling of the inverted resistivity data, $\rho t^{k}$ is the experimental apparent resistivity curve obtained from the forward modeling of the true resistivity model. The variable $N$ is amount of the resistivity data points.

\section{Inversion Results and Discussion}

In order to display the robustness of the GA inversion technique, three typical models with some ideal resistivity profile of geological relevance are chosen. The aim of our inversion is to estimate subsurface parameters, i.e., formation resistivity and thickness of different subsurface layers. The inversion results of three models presented below represent special cases in the application of the direct current resistivity technique.

\subsection{Model-1: Inversion of three-layer model}

For this model, we represent irregularly formation resistivity and thickness having within the high resistivity layer embedded by same conductive layers. This example was adopted from Sen et al., [5], and model consists of three layers with five model parameters (Table 1). For the execution of the GA program we adopt the search limits from Sen et al., [5] listed in Table 1.
Table 1: True model and search limits for Model-1

\begin{tabular}{|c|c|c|c|c|c|c|}
\hline \multirow{2}{*}{$\begin{array}{c}\text { Layer } \\
\text { Number }\end{array}$} & \multicolumn{2}{|c|}{ True Model-1 } & \multicolumn{4}{|c|}{ Resistivity and thickness limits } \\
\cline { 2 - 7 } & $\rho(\Omega . \mathrm{m})$ & $h(\mathrm{~m})$ & $\begin{array}{c}\rho^{\min } \\
(\Omega . \mathrm{m})\end{array}$ & $\begin{array}{c}\rho^{\max } \\
(\Omega . \mathrm{m})\end{array}$ & $\begin{array}{c}h^{\min } \\
(m)\end{array}$ & $\begin{array}{c}h^{\max } \\
(m)\end{array}$ \\
\hline $1^{\text {st }}$ layer & 10 & 10 & 5 & 15 & 1 & 20 \\
\hline $2^{\text {nd }}$ layer & 390 & 250 & 15 & 500 & 100 & 500 \\
\hline Half space & 10 & - & 1 & 20 & - & - \\
\hline
\end{tabular}

The experimental apparent resistivity curve of the model consists of 20 data points distributed logarithmically and was calculated using our forward modeling program code at points given by dotted line with a square shown in Fig. 1. White Gaussian noise was added to the experimental apparent resistivity in order to test the sensitivity of the inversion under field conditions. The results from GA inversion are illustrated by the solid line with squares in Figure 1, showing best match between the inverted and observed apparent resistivity curves, which suggests reliable layer parameters estimated by the GA inversion.

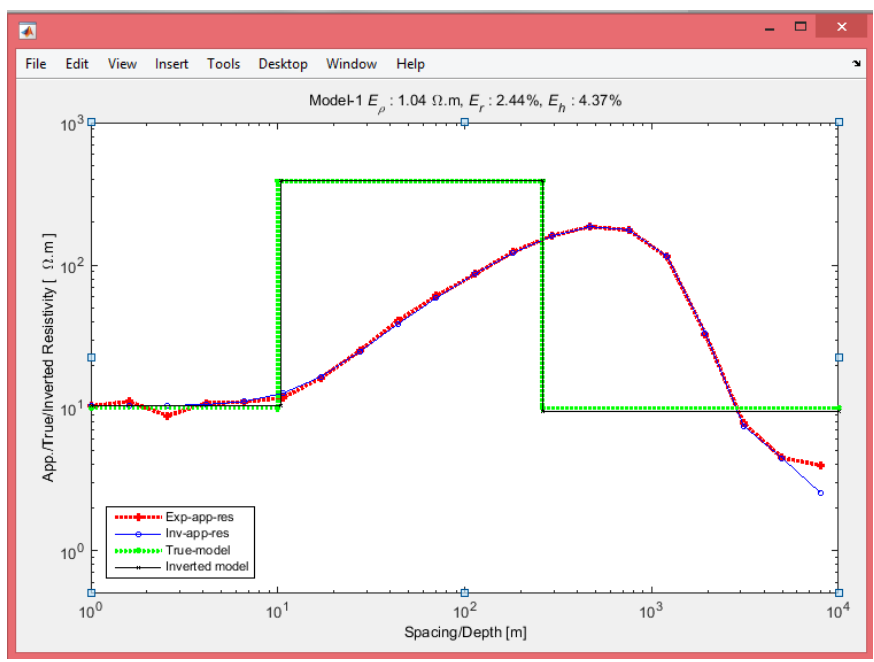

Figure 1: Apparent resistivity curves and inverted resistivity for Model-1

Accuracy of the inversion results is analyzed by quantifying estimated apparent resistivity curve errors and estimated parameter errors (e.g. resistivity value errors, $E_{r}$, and thickness error, $\left.E_{h}\right)$. Equation 8 was used to quantify the apparent resistivity curve errors $\left(E_{\rho}\right)$. Using GA optimization technique, apparent resistivity curve error $\left(E_{\rho}\right)$ is $1.04 \Omega . \mathrm{m}$. The resistivity value errors $\left(E_{r}\right)$ and thickness error $\left(E_{h}\right)$ are respectively quantified by using equations 5 and 6 . The $E_{r}$ and $E_{h}$ for GA inversion technique are $2.44 \%$ and $4.37 \%$, respectively.

Table 2: Inverted values for Model-1

\begin{tabular}{|c|c|c|c|c|}
\hline \multirow{2}{*}{$\begin{array}{c}\text { Number } \\
\text { Layer }\end{array}$} & \multicolumn{2}{|c|}{ Resistivity $(\Omega . m)$} & \multicolumn{2}{c|}{ Thickness $(\mathrm{m})$} \\
\cline { 2 - 5 } & GA & SA & GA & SA \\
\hline $1^{\text {st }}$ layer & $10.28 \pm 0.29$ & $9.72 \pm 0.89$ & $10.34 \pm 0.35$ & $9.72 \pm 0.89$ \\
\hline \multirow{2}{*}{$2^{\text {nd }}$ layer } & $390.21 \pm 0.19$ & $394.00 \pm$ & $252.41 \pm$ & $250.00 \pm$ \\
& & 13.60 & 2.42 & 9.31 \\
\hline Half space & $9.47 \pm 0.50$ & $10.10 \pm 0.75$ & - & - \\
\hline
\end{tabular}

Comparison of our estimated model parameters using GA with those of using SA (listed in Table 2) reveals that the results are similar. Note that Sen et al., [5] used noise free to their data while ours had $5 \mathrm{~dB}$ white noises added.

\section{Volume 4 Issue 11, November 2015}




\section{International Journal of Science and Research (IJSR) \\ ISSN (Online): 2319-7064}

Index Copernicus Value (2013): 6.14 | Impact Factor (2014): 5.611

Nevertheless, error values resulted from our inversion are smaller than those of them.

\subsection{Model-2: Inversion of five-layer model}

The example for this model was also taken from paper by Sen et al., [5] comprising five layers with nine model parameters. The search limits used in our GA inversion are also same as those used in SA by Sen et al., [5]. Detailed model parameters and search limits of the model are listed in Table 3.

Table 3: True model and search limits for Model-2

\begin{tabular}{|c|c|c|c|c|c|c|}
\hline \multirow{2}{*}{$\begin{array}{c}\text { Layer } \\
\text { Number }\end{array}$} & \multicolumn{2}{|c|}{ True Model-1 } & \multicolumn{4}{|c|}{ Resistivity and thickness limits } \\
\cline { 2 - 7 } & $\rho(\Omega . \mathrm{m})$ & $h(m)$ & $\begin{array}{c}\rho^{\min } \\
(\Omega . \mathrm{m})\end{array}$ & $\begin{array}{c}\rho^{\max } \\
(\Omega . \mathrm{m})\end{array}$ & $\begin{array}{c}h^{\min } \\
(m)\end{array}$ & $\begin{array}{c}h^{\max } \\
(m)\end{array}$ \\
\hline $1^{\text {st }}$ layer & 10 & 2 & 1 & 15 & 1 & 20 \\
\hline $2^{\text {nd }}$ layer & 50 & 15 & 15 & 500 & 100 & 500 \\
\hline $3^{\text {rd }}$ layer & 100 & 20 & & & & \\
\hline $4^{\text {th }}$ layer & 20 & 25 & & & & \\
\hline Half space & 400 & - & 1 & 20 & $\infty$ & $\infty$ \\
\hline
\end{tabular}

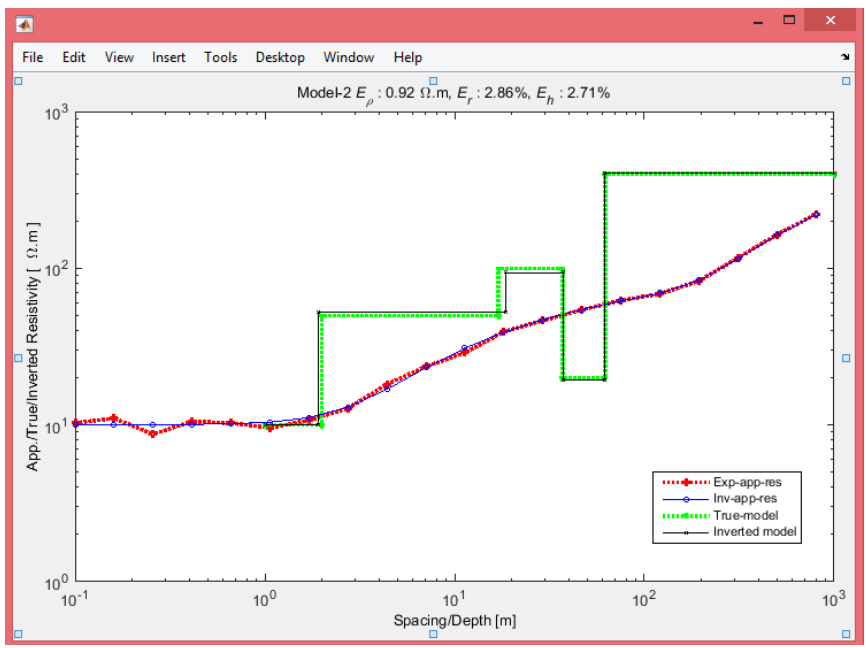

Figure 2: Apparent resistivity curves and inverted resistivity for Model-2

Execution results from our forward modeling code generated the experimental apparent resistivity curve consisting of 20 data points distributed logarithmically. $5 \mathrm{~dB}$ white Gaussian noises were added to the experimental apparent resistivity. The experimental and theoretical apparent resistivity curves, along with the true and inverted resistivity models are shown in Fig. 2. The curve of experimental apparent resistivity of the model is illustrated by dotted line with squares, while the theoretical apparent resistivity is resulted from our GA inversion is illustrated by solid line with a square in Fig. 2. It can be seen from this figure that the theoretical apparent resistivity curve matches well with the experimental one. For this model, the apparent resistivity curve error $\left(E_{\rho}\right)$ is 0.92 $\Omega . \mathrm{m}$. The resistivity value error $\left(E_{r}\right)$ and thickness error $\left(E_{h}\right)$ of the model are $2.86 \%$ and $2.71 \%$, respectively. The optimal subsurface layer parameters at the Model-2 obtained by the GA inversion technique and by the $\mathrm{SA}$ inversion are summarized in Table 4.
Table 4: Inverted values of model parameters for Model-2

\begin{tabular}{|c|c|c|c|c|}
\hline \multirow{2}{*}{$\begin{array}{c}\text { Number } \\
\text { Layer }\end{array}$} & \multicolumn{2}{|c|}{ Resistivity $(\Omega . m)$} & \multicolumn{2}{c|}{ Thickness $(\mathrm{m})$} \\
\cline { 2 - 5 } & GA & SA & GA & SA \\
\hline $1^{\text {st }}$ layer & $10.05 \pm 0.05$ & $10.00 \pm 0.77$ & $1.90 \pm 0.09$ & $1.94 \pm 0.40$ \\
\hline $2^{\text {nd }}$ layer & $52.55 \pm 2.67$ & $47.70 \pm 7.13$ & $16.63 \pm 1.81$ & $14.20 \pm 2.76$ \\
\hline $3^{\text {rd }}$ layer & $93.99 \pm 5.64$ & $\begin{array}{c}106.00 \pm \\
15.81\end{array}$ & $18.93 \pm 2.06$ & $19.40 \pm 4.16$ \\
\hline $4^{\text {th }}$ layer & $19.32 \pm 0.65$ & $20.60 \pm 7.18$ & $24.13 \pm 2.63$ & $26.20 \pm 9.20$ \\
\hline Half space & $403.81 \pm$ & $402.00 \pm$ & - & - \\
\hline
\end{tabular}

It can be seen from Table 4 that our inverted model parameters are close enough to the true ones, which in turn suggests reliable estimate of layer parameters by the GA optimization technique. The error values resulted from our inversion are smaller than those of Sen et.al., [5] in spite of our data in the inversion incorporating $5 \mathrm{~dB}$ white noises.

\section{Conclusion}

In this study, codes for 1D resistivity forward modeling and its inversion using the GA optimization technique have been developed under the MATLAB environment using version 8.4.0.150421 Release R2014b. MATLAB provides integrated functionality of computation and visualization, extensive numerical libraries, data visualization capabilities, and enables the user to interactively exploit data variables step by step during execution. MATLAB is no difficult to be used and employs a high-level programming language grammar, employs simple vector and matrix multiplication and it doesn't need memory dimensioning. The use of nonlinear optimization techniques such as the GA can be powerful and efficient inversion method to solve resistivity sounding data. Non linear inversion techniques are appropriate due to the fact that apparent resistivity values measured on the surface as a function of electrode separation have a nonlinear relationship to the subsurface model parameters, i.e., the resistivity and thickness of layer.

Our application of GA inversion implemented under the MATLAB environment for inverting synthetic resistivity sounding data has provided very significant inversion results with errors are smaller than the traditional techniques. From the successful inversions of the two synthetic models, we conclude that our GA optimization technique can be practically applied to resolve 1D layer parameters with reasonably low inversion error values. Analysis of the inversion results of this study show that the inverted resistivity values of the model parameter are very close to the true resistivity values. Inversion results from all synthetic models show small (less than $5 \%$ ) errors of resistivity and thickness for each layer. This indicates reliable estimates of subsurface parameter by the GA optimization.

\section{Acknowledgements}

This research is supported by Directorate of Higher Education Indonesia with scheme of international research collaboration and scientific publication 2013. We also would like to thank Universitas Halu Oleo in Kendari, Southeast Sulawesi Indonesia and School of Physics Universiti Sains Malaysia for collaboration and financial support.

\section{Volume 4 Issue 11, November 2015}




\section{International Journal of Science and Research (IJSR) \\ ISSN (Online): 2319-7064}

Index Copernicus Value (2013): 6.14 | Impact Factor (2014): 5.611

\section{References}

[1] Muiuane, E.A., Pedersen, L.B., Automatic 1D interpretation of DC resistivity sounding data. Journal of Applied Geophysics 42, 35-45, 1999.

[2] Davis, P.A., Interpretation of resistivity sounding data: computer programs for solution to the forward and inverse problems. Information Circular 17, Minnesota Geological survey, 1979.

[3] Slaoui, F.H., Georges, S., Lagace, P.J., Do, X.D., The inverse problem of Schlumberger resistivity sounding measurement by ridge regression. Electric Power System Research 67, 109-114, 2003.

[4] Vedanti, N., Srivastava, R.P., Sagode, J., Dimri, V.P., An efficient 1D Occam's inversion algorithm using analytically computed first-and second-order derivatives for DC resistivity soundings. Computers \& Geosciences 31, 319-328, 2005.

[5] Sen, M.K., Bhattacharya, B.B., Stoffa, P.L., Nonlinear inversion of resistivity sounding data. Geophysics 58(4), 496-507, 1993.

[6] Nagai, K., O’Neill, A., Sanada, Y., Ashida, Y., Genetic algorithm inversion of Rayleigh wave dispersion from CMPCC gathers over shallow fault model. Journal of Environmental and Engineering Geophysics 10 (3), 135-150, 2005.

[7] Song, X.H., Gu, H.M., Utilization of multimode surface wave dispersion for characterizing roadbed structure. Journal of Applied Geophysics 63(2), 59-67, 2007.

[8] Hamimu, L., Safani, J., Nawawi, M., Improving the accurate assessment of a shear-wave velocity reversal profile using joint inversion of the effective Rayleigh wave and multimode Love wave dispersion curves. Near Surface Geophysics 9(1), 1-14, 2011

[9] Hamimu, L., Nawawi, M., Safani, J., Utilization of multimode Love wave dispersion curve inversion for geotechnical site investigation. Journal of Geophysics and Engineering 8(2), 341-350, 2011.

[10] Jha, M.K., Kumar, S., Chowdhury, A., Vertical electrical sounding survey and resistivity inversion using genetic algorithm optimization technique. Journal of Hydrology 359, 71-87, 2008.

[11] Kohlbeck, F., Mawlood, D., Computer program to calculate resistivities and layer thicknesses from Schlumberger soundings at the surface, at lake bottom and with two electrodes down in the subsurface. Computers \& Geosciences 35, 1748-1751, 2009.

[12] Lee, S.K., Kim, H.J., Song, Y., Lee, C.K., MT2DInvMATLAB-A program in MATLAB and FORTRAN for two-dimensional Magnetoteluric inversion. Computers \& Geosciences 35, 1722-1734, 2009.

[13] Ghosh, D.P., Inverse filter coefficients for the computation of apparent resistivity standard curves for horizontally stratified earth. Geophysical Prospecting 19, 769-775, 1971.

[14] Sen, M.K., and Stoffa, P.L., Global optimization methods in geophysical inversion (vol 4: series ed): A J Berkhout, Amsterdam: Elsevier, 125-244, (1995).

[15] Tsutsui, S., Fujimoto, Y., Ghosh, A., Forking GAs: GAs with search space division schemes. Evolutionary
Computation 5(1), 61-80, 1997.

[16] Sivanandan, S.N., and Deepa, S.N., Introduction to Genetic Algorithms, Springer Berlin Heidelberg New York, 50-56, 2008.

[17] Yamanaka, H., Ishida, H., Application of genetic algorithms to an inversion of surface wave dispersion data. Bulletin of the Seismological Society of America 86(2), 436-444, 1996.

\section{Author Profile}

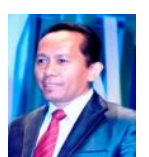

La Hamimu received the B.Sc. from Math and Natural Sciences Faculty, Gadjah Mada University (in Physics) and Master Program Degree (MT) in Geophysics from Bandung Institute of Technology Indonesia in 1997 and 2003, respectively. During 2008-2011, he received Ph.D degree in Near Surface Geophysics and Geophysical Computation Modelling in School of Physics, Universiti Sains Malaysia. 